\title{
Ethanol suppression of peripheral blood mononuclear cell trafficking across brain endothelial cells in immunodeficiency virus infection
}

This article was published in the following Dove Press journal:

HIVIAIDS - Research and Palliative Care

28 January 2010

Number of times this article has been viewed

\author{
Lola C Hudson' \\ Brenda A Colby' \\ Rick B Meeker ${ }^{2}$ \\ 'Department of Molecular \\ Biosciences, College of Veterinary \\ Medicine, North Carolina State \\ University, Raleigh, NC, USA; \\ ${ }^{2}$ Department of Neurology, School \\ of Medicine, University of North \\ Carolina at Chapel Hill, Chapel Hill, \\ NC, USA
}

Correspondence: Lola C Hudson NCSU-CVM, 4700 Hillsborough St. Raleigh, NC 27606, USA

$\mathrm{Tel}+19195136306$

$\mathrm{Fax}+19195136465$

Email lola_hudson@ncsu.edu

\begin{abstract}
Earlier studies suggested that the combination of alcohol use and immunodeficiency virus infection resulted in more severe neurologic disease than either condition individually. These deleterious interactions could be due to increased immune cell and virus trafficking or may result from interactions between ethanol and human immunodeficiency virus (HIV)-associated toxicity within the brain. To determine the extent to which increased trafficking played a role, we examined the effect of ethanol on the migration of different peripheral blood mononuclear cell (PBMCs) subsets across a brain endothelial cell monolayer. We utilized combinations of feline brain endothelial cells with astrocytes, and/or microglia with either acute exposure to $0.08 \mathrm{~g} / \mathrm{dL}$ ethanol, a combination of ethanol and feline immunodeficiency virus (FIV), or FIV alone. Adherence of PBMCs to endothelium was increased in all combinations of cells with the addition of ethanol. Despite increased PBMC adhesion with ethanol treatment, transmigration of B cells, monocytes, CD4 T cells and CD8 T cells was not increased and was actually decreased in the presence of astrocytes. Expression of three common adhesion molecules, intercellular adhesion molecule-1 (ICAM1), ICAM2, and vascular cell adhesion molecule, was unchanged or slightly decreased by ethanol. This indicated that although adherence is increased by ethanol it is not due to an increased expression of adhesion molecules. RANTES, MIP1 $\alpha$, MIP1 $\beta$, and MCP-1 mRNA expression was also studied in brain endothelial cells, astrocytes and microglia by reverse transcriptase-polymerase chain reaction. Ethanol treatment of astrocytes resulted in modest changes of message while FIV caused 7-92-fold increases. The combination of ethanol and FIV reversed the large increase in RANTES and MIP1 $\alpha$ message in astrocytes but increased MIP1 $\beta$ and MCP to 20-38-fold over control cells. Thus, modest concentrations of alcohol do not directly influence immune cell trafficking at the endothelium but may exert more complex effects on chemokine expression from astrocytes when combined with FIV.
\end{abstract}

Keywords: HIV, PBMC, adhesion molecule, chemokine

\section{Introduction}

The incidence of human immunodeficiency virus (HIV) and HIV-associated neurologic disease was reported at higher rates in alcohol abusers by some authors. ${ }^{1-5}$ However, it has been unclear whether or not the increase in central nervous system (CNS) infection and pathogenesis seen with alcohol consumption is due to a greater prevalence of high risk behaviors, more efficient penetration of human immunodeficiency virus (HIV) into the brain or synergistic pathological interactions. Experimental studies that were designed to explore the interactions of alcohol with HIV infection and pathogenesis have provided mixed results. Studies with simian immunodeficiency virus (SIV) showed that alcohol-exposed macaques had higher levels of cerebrospinal fluid (CSF) virus, plasma viremia, and more severe alterations in peripheral blood mononuclear 
cells (PBMC) subset numbers. ${ }^{6,7}$ An increased infection of PBMCs is seen after treatment with ethanol suggesting that these cells may be capable of delivering a greater viral load to target tissues. ${ }^{8}$ In addition, the effects of ethanol on endothelium may modulate PBMC adhesion and trafficking. ${ }^{9}$ Both increases and decreases in adhesion were reported in peripheral endothelium. ${ }^{9-11}$ Alterations at the endothelium of vessels as suggested by Sacanella and colleagues ${ }^{12}$ may allow increased trafficking of PBMCs. If this change is also occurring at the brain endothelium, it may allow preferential trafficking of infected cells or increased trafficking of all PBMCs with greater access to the brain parenchyma. Yet other reports, focusing more on epidemiological studies, ${ }^{13,14}$ suggested that earlier epidemiological data was misinterpreted and that an association between alcohol and increased HIV pathogenesis was not present.

The feline immunodeficiency virus (FIV) model allows assessment of the effects of ethanol in a complete in vitro blood-brain barrier system that includes all potential cell targets (endothelial cells, astrocytes, and microglia). Using this model, we sought to determine if ethanol influenced PBMC trafficking across brain endothelium under normal conditions and in the presence of FIV. Feline brain endothelial cells were cultured in the upper chamber of in vitro cell culture inserts and combined with astrocytes and/or microglia in the lower chamber to mimic the normal brain environment. The effect of ethanol on adherence of PBMCs to the endothelial cell monolayer and transmigration of B cells, CD4 $\mathrm{T}$ cells, CD8 T cells, and monocytes was measured.

\section{Methods and materials}

\section{Cells and culture system}

The source of tissue for cell cultures, isolation methods for CNS cells and PBMCs, characterization of these cells, presence of endothelial cell tight junctions, source of FIV, in vitro cell culture system, seeding protocols, immunocytochemistry of trafficked cells, and automated cell counting software used in these experiments were previously described in detail. ${ }^{15}$ Two independent experiments with two or three replicates were used to produce an number of five or six for each experiment.

Briefly, the brains of deceased feline fetuses were removed, washed, and processed for culture of brain endothelial cells (BECs), astrocytes, and microglia. The purity of these primary cultures was determined using specific cell markers and ranged from $>80 \%$ for BECs to $>95 \%$ for astrocytes and microglia. BECs were subcultured a maximum of six passages and cells from each passage were frozen for later use. Astrocytes were subcultured once and microglia were not subcultured.

Endothelial cells were seeded at $5 \times 10^{5}$ /insert onto a transwell insert membrane coated with collagen and fibronectin and containing $3 \mu \mathrm{m}$ pores. Each transwell insert was placed into a well of a 24-well plate which constituted the lower chamber. BECs were allowed to grow to confluence, which was generally 2-3 days. For experiments that would ultimately include treated astrocytes or microglia, the appropriate cell type(s) was seeded at $1 \times 10^{5}$ cells into the lower chamber. Newly seeded BEC inserts were placed into the wells to allow exposure of BECs to any secreted factors normally produced by the accessory cells. A parallel set of plates with astrocytes and/or microglia were prepared identically. Brain endothelial cells were always grown in the upper chamber and were treated alone or in combination with astrocytes, and/or microglia in the lower chamber giving rise to four culture conditions: 1) endothelium (BECs), 2) endothelium + astrocytes $(\mathrm{BECs}+\mathrm{A}), 3)$ endothelium + microglia (BECs+MG) and 4) endothelium + astrocytes + microglia (BECs+A\&MG). Cells received either no treatment, ethanol, FIV, or ethanol and FIV treatment (see Table 1). On day 2 of co-culture, astrocytes or microglia (without endothelial cell monolayer inserts) were exposed to ethanol, ethanol and FIV- $\mathrm{NCSU}_{1}$, or FIV-NCSU ${ }_{1}$ in fresh medium for 24 hours. On day 3, the astrocytes or microglia were washed three times, and then the inserts with confluent BECs were transferred to the treated wells. To determine the direct effect of FIV and ethanol on the BECs, the same treatment conditions were applied to the confluent endothelial cell layer for 24 hours. The BECs were washed three times prior to addition of PBMCs. After the treatment of BECs, astrocytes, or microglia and the wash steps, $1 \times 10^{5} \mathrm{PBMCs}$ were added to the upper chamber of each well for 24 hours. Cells in control configurations received fresh medium in lieu of treatment and wash steps.

\section{Treatment with ethanol and FIV}

Ethanol was added to medium at a concentration of $101.4 \mu \mathrm{L}$ of $100 \%$ ethanol per $100 \mathrm{~mL}$ of medium to produce the equivalent of $80 \mathrm{mg} / \mathrm{dL}$ blood alcohol level (0.08\%); the legal basis for drunk driving in the United States (http://www. alcoholcontrols.com/lebaclibyst.html). In constructs where astrocytes or microglia were present, only the cells in the lower chamber received treatment (ie, not BECs). After washing treated cells three times, inserts with confluent untreated BECs were transferred to wells containing astrocytes and/or microglia. These studies of ethanol effects were run in parallel 
Table I Configuration (ie, combinations) of cells and treatment regimens

\begin{tabular}{|c|c|c|c|c|c|}
\hline & BECs & Astrocytes & Microglia & $\begin{array}{l}\text { Astrocytes } \\
\text { and microglia }\end{array}$ & Replicates (n) \\
\hline \multirow[t]{4}{*}{ No treatment } & $x-$ & & & & 5 \\
\hline & $x-$ & $x-$ & & & 5 \\
\hline & $x-$ & & $x_{-}$ & & 5 \\
\hline & $x-$ & & & $X_{-}$ & 5 \\
\hline \multirow[t]{4}{*}{ Ethanol treatment } & $\mathrm{X}+$ & & & & 6 \\
\hline & $x_{-}$ & $x_{+}$ & & & 6 \\
\hline & $x-$ & & $X_{+}$ & & 6 \\
\hline & $x_{-}$ & & & $\mathrm{X}+$ & 6 \\
\hline \multirow[t]{4}{*}{ Ethanol and FIV treatment } & $x_{+}$ & & & & 6 \\
\hline & $x-$ & $x_{+}$ & & & 6 \\
\hline & $x_{-}$ & & $\mathrm{X}_{+}$ & & 6 \\
\hline & $x-$ & & & $x_{+}$ & 6 \\
\hline \multirow[t]{4}{*}{ FIV treatment } & $x_{+}$ & & & & 5 \\
\hline & $x-$ & $x_{+}$ & & & 5 \\
\hline & $x_{-}$ & & $X_{+}$ & & 5 \\
\hline & $x_{-}$ & & & $x_{+}$ & 5 \\
\hline
\end{tabular}

Notes: X, cell type present in culture configuration; -, cell type present but did not receive ethanol and/or FIV treatment; +, cell type cocultured with ethanol and/or FIV treatment.

Abbreviations: FIV, feline immunodeficiency virus; BEC, brain endothelial cells.

with other studies of PBMC trafficking. ${ }^{15}$ The FIV treatment was accomplished by adding $50 \mu \mathrm{L}$ of a diluted $\mathrm{NCSU}_{1}$ strain of FIV to a final concentration of $5 \times 10^{4} \mathrm{TCID}_{50} / 100 \mu \mathrm{L} .{ }^{16}$ The normal control cultures and the FIV control cultures reported here represent a subset of the controls previously summarized..$^{15}$

\section{Peripheral blood mononuclear cells}

For all experiments, anticoagulated blood was obtained from normal, specific pathogen-free cats. PBMCs were isolated on Percoll gradient and stimulated with $2 \mu \mathrm{g} / \mathrm{mL}$ concanavalin A overnight. These cells were then labeled with $5 \mu \mathrm{M}$ Cell Tracker Orange ${ }^{\circledR}$ (Molecular Probes, Invitrogen, Carlsbad, CA, USA) per the manufacturer's instructions. PBMCs were then added $\left(1 \times 10^{5} /\right.$ well $)$ to the upper chamber of the cell culture inserts. After 24 hours, the inserts were gently washed to remove nonadherent cells and mounted onto slides with the endothelial cell layer facing up (simulating the vascular lumen). Fluorescent PBMCs adhered to the surface of the BEC monolayer were counted in five fields (field $=0.2515 \mathrm{~mm}^{2}$ ) for each membrane using Metamorph $^{\circledR}$ imaging software (Universal Imaging Corp. Westchester, PA, USA). Fluorescent objects were counted after filtering by size to only measure objects in the size range of white blood cells. Counts were averaged within each insert to provide a single value for each insert. The various configurations and number of replicates analyzed are summarized in Table 1.

The medium from the lower chambers of configurations including transmigrated PBMCs was collected, evenly divided and cytospun onto two slides. The cells were then stained for CD3, CD4, CD8, B cell, or monocyte markers and manually counted as described previously. ${ }^{15}$ Techniques for double immunocytochemistry reduced the number of cytospins needed to count all PBMC subsets studied with each configuration and increased sample number.

\section{Western blot analysis}

Brain endothelial cells were grown to confluence in Iscove's medium (Mediatech, Manassas, VA, USA) containing 10\% fetal bovine serum (FBS; Mediatech, Manassas, VA, USA), $20 \mu \mathrm{g} / \mathrm{mL}$ endothelial cell growth supplement (Collaborative Research, Bedford MA), 6 U/mL heparin (Sigma, St. Louis, MO, USA), $25 \mu \mathrm{g} / \mathrm{mL}$ gentamicin (Mediatech, Manassas, VA, USA), and $0.025 \mu \mathrm{g} / \mathrm{mL}$ amphotericin B (Mediatech, Manassas, VA, USA). Fresh medium was combined 1:1 $(\mathrm{v} / \mathrm{v})$ with filtered conditioned medium from earlier BEC cultures. Fresh medium containing ethanol at $101.4 \mu \mathrm{L}$ of 
$100 \%$ ethanol $/ 100 \mathrm{~mL}$ of medium $(80 \mathrm{mg} / 100 \mathrm{dL}), \mathrm{FIV}$ at MOI of 0.5 , or ethanol (same dose) plus FIV (same dose) were added to these cultures for 24 hours. Control cultures received fresh medium with no added ethanol or FIV. Cells were sonicated in buffer (50 mM Tris-HCl, $150 \mathrm{mM}$ $\mathrm{NaCl}, 1 \mathrm{mM}$ EDTA, pH 8.0) with 1\% fresh stock cocktail of protease inhibitors (final concentrations: pepstatin A $50 \mu \mathrm{g} / \mathrm{mL}$, chymostatin $50 \mu \mathrm{g} / \mathrm{mL}$, leupeptin $50 \mu \mathrm{g} / \mathrm{mL}$, and aprotinin $100 \mu \mathrm{g} / \mathrm{mL})$. Protein concentrations were determined by Bradford assay. Samples and controls were boiled for two minutes prior to loading. Positive control, negative control, and sample wells were loaded with $45 \mu \mathrm{g}$ of protein in a denaturing SDS buffer. Samples were subjected to electrophoresis in a $7.5 \%$ polyacrylamide gel, and then transferred to polyvinylidene difluoride membrane overnight at $4{ }^{\circ} \mathrm{C}$ and $40 \mathrm{~V}$. The membrane was blocked in 5\% skim milk buffer overnight, incubated with primary antibody for intercellular adhesion molecule-1 (ICAM1), ICAM2, or vascular cell adhesion molecule (VCAM) (Table 2) for one hour at room temperature, then incubated with horseradish peroxidase (HRP)-conjugated secondary antibody for one hour at room temperature. Peroxidase activity was detected by chemiluminescence (Pierce Biotechnology, Rockford IL, USA). Bands were imaged using a bioimaging system and Labworks ${ }^{\circledR}$ software (UVP, Upland, CA, USA). The membranes were then stripped using a commercial product (Pierce Biotechnology) and reprobed for actin with an HRP-conjugated secondary antibody and chemiluminescence as described above as a loading control. For the resulting figure, blot images were optimized by adjusting contrast to view bands and pertinent sample lanes were digitally placed adjacent to each other.

\section{Real time reverse transcriptase- polymerase chain reaction (RT-PCR)}

Enriched feline endothelial cells, astrocytes, or microglia were grown in tissue culture flasks. Media containing ethanol, etha-

Table 2 Western blot reagents

\begin{tabular}{lll}
\hline Primary & Secondary & Substrate** \\
Ab*II:I00 & Ab*II:10,000 & \\
\hline ICAM I & Bovine anti-goat-HRP & Chemiluminescence \\
ICAM 2 & Goat anti-rabbit-HRP & Chemiluminescence \\
VCAM & Bovine anti-goat-HRP & Chemiluminescence \\
Actin & Bovine anti-goat-HRP & Chemiluminescence \\
\hline
\end{tabular}

Notes: *Santa Cruz Biotechnology, Santa Cruz, CA, USA; **Pierce Biotechnology kit, Rockford, IL, USA.

Abbreviations: HRP, horseradish peroxidase; ICAM, intercellular adhesion molecule; VCAM, vascular cell adhesion molecule. nol with FIV, FIV, and no treatment was applied for 24 hours to three independent studies. Ethanol was added to medium at a final concentration of $80 \mathrm{mg} \%$. FIV was applied at an MOI of 0.5 . At the end of treatment, cells were collected and archived in RNAprotect Cell Reagent (Qiagen, Valencia, CA, USA). After thawing, cells were processed for RNA per manufacturer's instructions (RNeasy Plus Mini Kit; Qiagen). cDNA was synthesized with integrated removal of genomic DNA contamination using $85 \mathrm{ng}$ of RNA per reaction (QuantiTect Reverse Transcription Kit; Qiagen). RT-PCR quantification of cDNA was performed on the Icycler IQ (BioRad, Hercules, CA, USA) in specified reaction mixtures including primers (Table 3) for feline RANTES, MIP1 $\alpha$, MIP1 $\beta$, MCP-1, and housekeeping G3PDH genes (QuantiTect SYBR Green PCR Kit; Qiagen). Negative controls included individual reactions containing each primer without the addition of cDNA; positive controls included reactions containing each primer with the addition of cDNA derived from feline PBMCs. Duplicate wells for each reaction step were performed and results were averaged. Fold increases and decreases were calculated using the method of Winer and colleagues. ${ }^{17}$

\section{Statistical analysis}

The absolute numbers of adherent and transmigrating PBMC subsets was variable from run to run resulting in a nonnormal distribution. Initially a Kruskal-Wallis analysis $(P<0.05)$ of each cell configuration (eg, BECs configuration with four treatment options) and of each treatment group (eg, untreated, ethanol-treated, ethanol- and FIV-treated, and FIV-treated BECs) was used. This was followed by Mann-Whitney analysis of data pairs $(P<0.05)$ to further evaluate changes in adherence of PBMCs and transmigration within each PBMC subset (eg, CD4 t cell transmigration

Table 3 Primer sequences for selected feline cytokines and housekeeping genes

\begin{tabular}{|c|c|c|}
\hline \multirow[t]{2}{*}{ Fe RANTES } & A: 5'-GCC/TCC/GCA/TCC/CCA/TAT/GCC & $179 \mathrm{bp}$ \\
\hline & B: 5'-CCC/ATT/TCT/TCT/GTG/GGT/TGG & \\
\hline \multirow[t]{2}{*}{ Fe MIPI $\alpha$} & A: 5'-CTC/GCC/ATC/CTC/CTC/TGC/ACC & $258 \mathrm{bp}$ \\
\hline & B: 5'-GGC/ACT/CAG/CTC/CAA/GTC/AG & \\
\hline \multirow[t]{2}{*}{ Fe MIPI $\beta$} & A: 5'-CTC/CTT/GTG/CTA/GTG/GCT/GCC & $200 \mathrm{bp}$ \\
\hline & B: 5'-GTC/ACT/GGG/GTT/AGC/ACA/GAC & \\
\hline \multirow[t]{2}{*}{$\mathrm{Fe} \mathrm{MCPI}$} & A: 5'-CTG/TGC/CTG/CTG/CTC/ATA/GCA/GC & $189 \mathrm{bp}$ \\
\hline & B: 5'-GGT/CTT/GAA/GAT/CAC/AGC/TTC & \\
\hline \multirow[t]{2}{*}{ G3PDH } & $\mathrm{A}: 5^{\prime}-\mathrm{CCT} / \mathrm{TCA} / \mathrm{TTG} / \mathrm{ACC} / \mathrm{TCA} / \mathrm{ACT} / \mathrm{ACA} / \mathrm{T}$ & $400 \mathrm{bp}$ \\
\hline & B: 5'-CCA/AAG/TTG/TCA/TGG/ATG/ACC & \\
\hline
\end{tabular}

Abbreviations: RANTES, regulated upon activation, normal T expressed and presumably secreted; MIP, macrophage inflammatory protein; MCP, macrophage chemoattractant protein; G3PDH, glyceraldehyde 3-phosphate dehydrogenase. 
associated with untreated BECs vs ethanol-treated BECs or CD8 $T$ cell transmigration associated with ethanol-treated $\mathrm{BECs}+\mathrm{A}$ and ethanol-treated BECS+MG) (GraphPad Prism ${ }^{\circledR}$ software; GraphPad, La Jolla, CA, USA).

For RT-PCR, untreated cells were indexed to a value of $1^{17}$ and relative increases and decreases were calculated for each experimental condition. Scores $>1.96$ standard error units from the controls were considered significant $(P<0.05)$. Treated groups were analyzed with each other using Kruskal-Wallis analysis $(P<0.05)$ and Mann-Whitney analysis of data pairs $(P<0.05)$.

\section{Results}

\section{Effect of ethanol on PBMC adherence}

The adherence of PBMCs was significantly increased after treatment of endothelial cells, astrocytes, or microglia with ethanol or with ethanol and FIV in all combinations of cells (Figure 1) compared to untreated cells and FIV treatment. FIV treatment resulted in a small but significant increase in adherence to the endothelium in the presence of astrocytes
(Figure 1), which was reversed with the addition of microglia. The effect of ethanol was not influenced by the presence of astrocytes or microglia (light gray bars).

\section{Effect of ethanol on PBMC transmigration}

Although the initial results supported increased adherence under ethanol treatment, transmigration of PBMCs did not follow the same trend. Endothelial cells with astrocytes had a relatively high level of trafficking across all PBMC subsets under normal conditions. Ethanol treatment decreased PBMC transmigration in this treatment group across all PBMC subsets. Endothelial cells with astrocytes also had a relatively high PBMC transmigration with FIV treatment, which was reversed by concurrent ethanol treatment. In Figures 2A-D, each cell subset was analyzed and the effect of treatments was assessed for each configuration.

For CD4 T cells (Figure 2A), there were no significant differences in transmigration within a particular configuration of CNS cells due to wide biologic variation. Only the ethanol and FIV treatment group in the configuration with

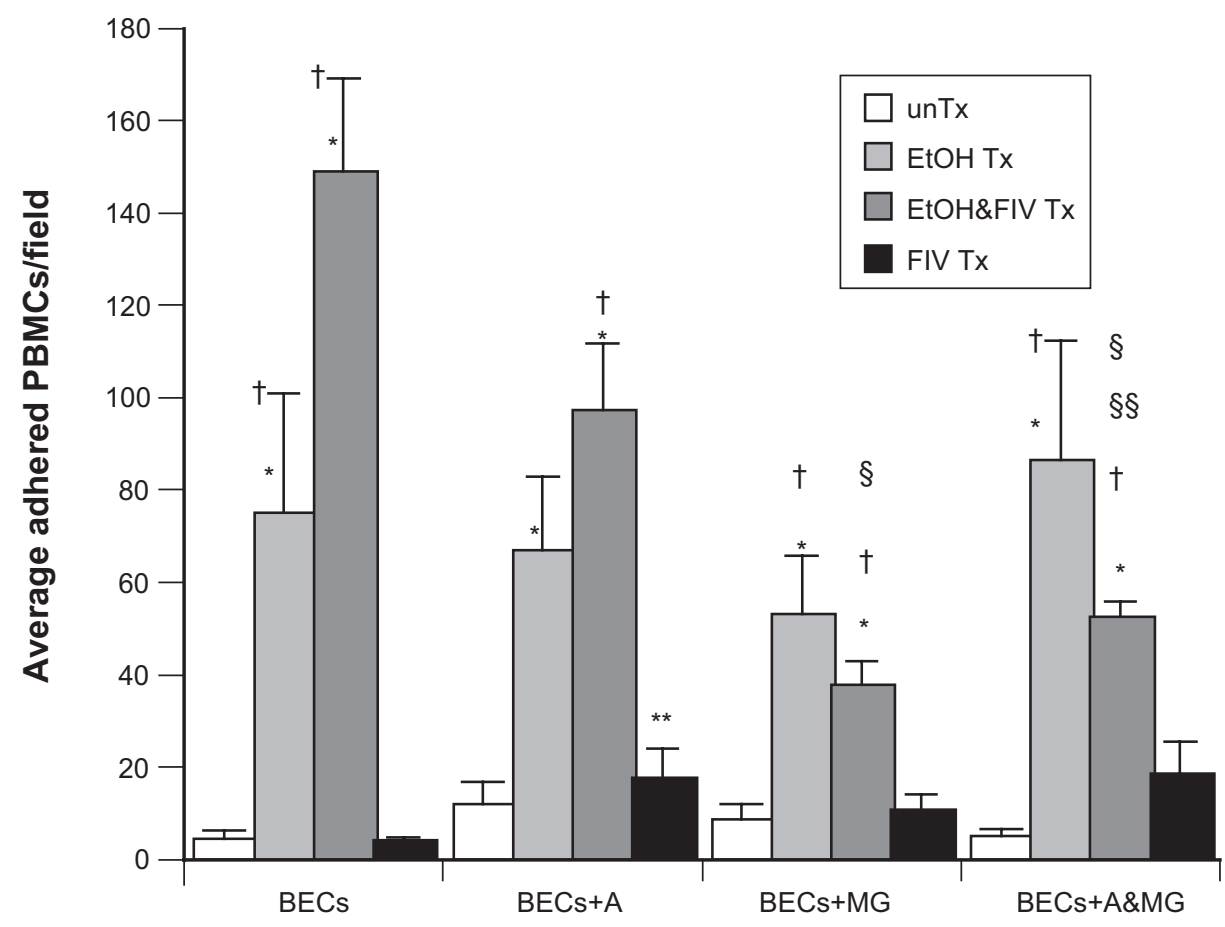

Figure I Peripheral blood mononuclear cells (PBMC) adherence after no treatment (unTx), ethanol (EtOH Tx) treatment, EtOH and feline immunodeficiency virus (FIV) treatment (EtOH\&FIV Tx) or FIV treatment (FIV Tx) of endothelial cells, astrocytes, and/or microglia. Astrocytes and/or microglia in the lower chamber of an in vitro cell culture insert system were treated or sham treated for 24 hours, and washed.Then inserts with confluent brain endothelial cells (BECs) were added to the well and cocultured with PBMCs for 24 hours. In the absence of astrocytes and microglia, BECs received the treatment or control medium instead, and then were cocultured with PBMCs. Error bars reflect standard error of the mean. Ethanol treatment showed dramatic, significant increases in mean adherence compared to untreated cells with all configurations of cells as did ethanol and FIV treatment $(* P<0.05)$. The ethanol or ethanol and FIV treatment was significantly increased over FIV treatment within most configurations of cells $\left({ }^{\dagger} P<0.05\right)$. For the treatment group receiving ethanol and FIV (dark gray bar) among the different configurations, those including microglia were significantly decreased relative to configurations without microglia $\left({ }^{(P}<0.05\right)$. Additionally, in this treatment group, the configuration with BECs, astrocytes and microglia was significantly increased over the configuration with BECs and microglia ( $(\$ \$ P<0.05)$.

Abbreviations: $+A$, astrocytes; +MG, microglia. 


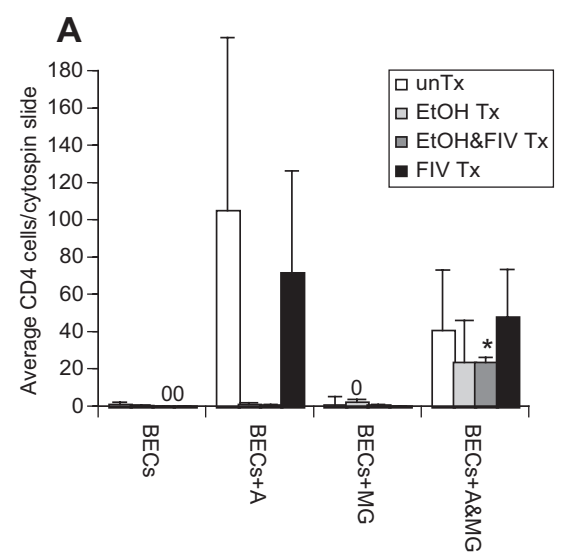

$\mathbf{B}$
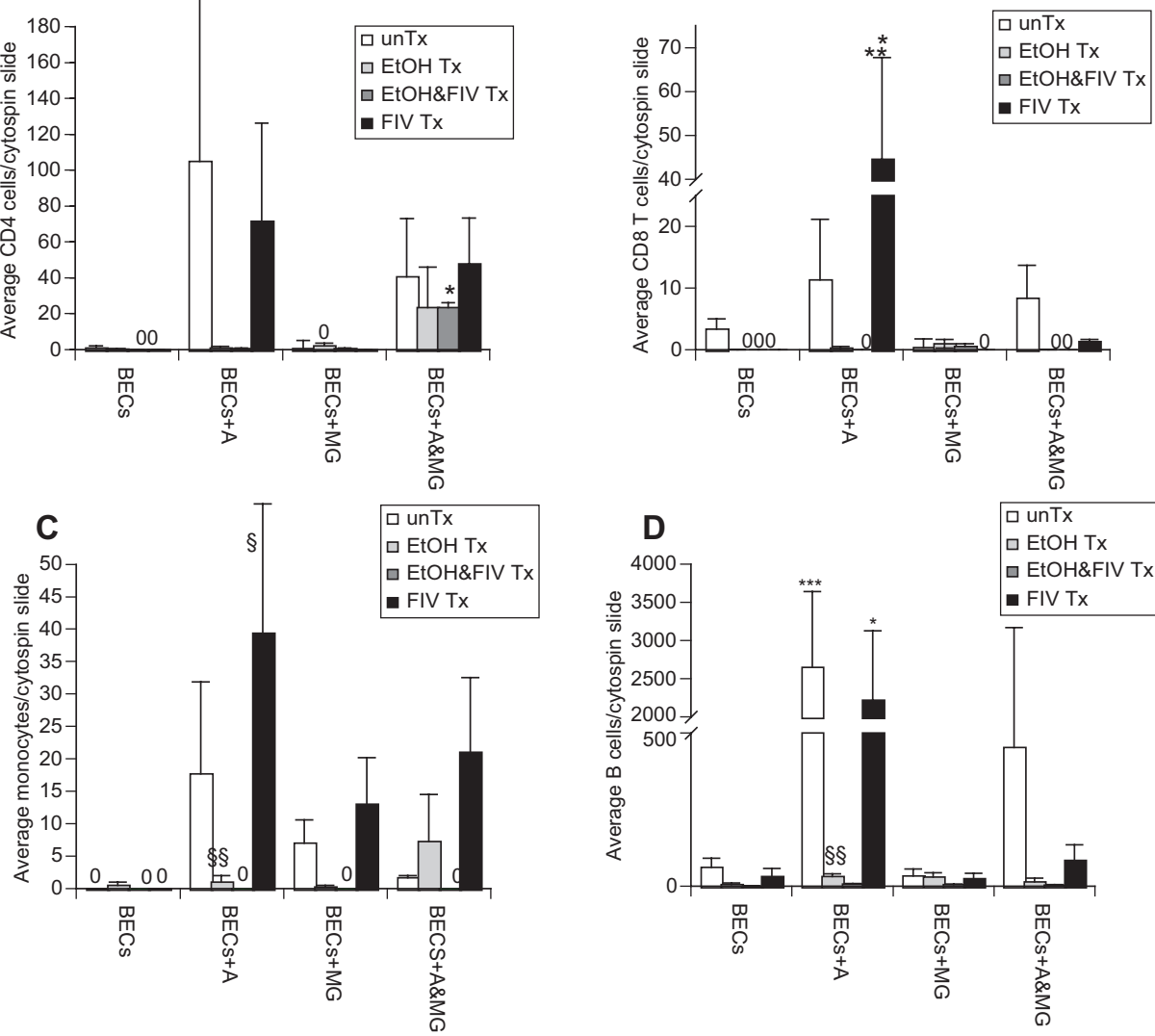

Figure 2 Peripheral blood mononuclear cells (PBMC) subset transmigration after treatment of brain endothelial cells (BECs), astrocytes (+A), and/or microglia (+MG) with ethanol (EtOH Tx), ethanol and FIV (EtOH\&FIV Tx), FIV (FIV Tx) or no treatment (unTx). CD4 T cells, CD8 T cells, monocytes, B cells were collected from the lower chamber of the cell culture insert system and cytospins were produced. Immunocytochemistry was used to identify each subset and slides were manually counted. Error bars reflect standard error of the mean. For all subsets of PBMCs, the inclusion of ethanol in the treatment in the BECs+A configuration yielded a decrease in transmigration. A) For CD4 T cells: In the EtOH and FIV treatment group, the configuration of BECs+A\&MG was significantly increased over all other cell configurations receiving the same treatment $(* P<0.00 I)$. B) For CD8 T cells: In the FIV treatment group, the BECs+A significantly increased transmigration compared to other treatment groups with the same cell configuration (**P < 0.05). In addition, the FIV-treated cells of this (BECs+A) configuration had significantly increased CD8+ T cell transmigration compared to all other cell configurations with FIV treatment $(* P<0.00 I)$. C) For monocyte cells: In the BECs+A configuration, ethanol and ethanol+FIV treatments significantly decreased transmigration compared to FIV treatment $(\$ \$ P<0.0 I)$. The FIV-treated cells of this $(B E C s+A)$ configuration had significantly increased monocyte transmigration compared to the configuration of BECs alone $(\S P<0.0 \mathrm{I})$. D) For $B$ cells: In the BECs+A configuration, ethanol treatment significantly decreased transmigration compared to untreated cells and FIV treatment $\left({ }^{\$} \mathrm{P}<0.00 \mathrm{I}\right)$. The untreated cells of this $(\mathrm{BEC}+\mathrm{A})$ configuration had significantly increased $\mathrm{B}$ cell transmigration compared to the untreated $\mathrm{BEC}$ configuration, and to the untreated BECs+MG configuration (***P $<0.001)$. The FIV-treated cells of this $(B E C s+A)$ configuration had significantly increased $B$ cell transmigration compared to all other configurations of cells with FIV treatment $(* P<0.0$ I $)$.

Abbreviation: FIV, feline immunodeficiency virus.

endothelial cells, astrocytes and microglia, was significantly increased over other configurations of cells.

CD8 $\mathrm{T}$ cell transmigration (Figure 2B), was increased within the BECs+A configuration. The FIV-treated cells were significantly increased over untreated, ethanol-treated, and ethanol- and FIV-treated BECs+A. No effect of ethanol was seen. In addition the BECs+A configuration with FIV treatment was significantly increased over all other configurations receiving FIV treatment.

Monocyte transmigration (Figure 2C), was significantly decreased in the presence of ethanol within the configuration of BECs+A compared to FIV treatment. FIV treatment significantly increased transmigration of these cells between $\mathrm{BECs}$ and BECs+A configurations.
B cell transmigration (Figure 2D) was increased with $\mathrm{BEC}+\mathrm{A}$ relative to BECs alone for both untreated and FIV-treated cultures. Ethanol suppressed transmigration of both the untreated cells and the FIV-treated cells.

The effect of ethanol on PBMC trafficking was supported by a failure to see increases in three common adhesion molecules. Western blot analysis of intercellular adhesion molecule-1 (ICAM1), ICAM2, and vascular cell adhesion molecule (VCAM) expression (Figure 3) showed that ethanol had no significant effect on adhesion molecule expression in the endothelial cells.

Changes in mRNA for four chemokines (RANTES, (regulated upon activation, normal $\mathrm{T}$ expressed and presumably secreted), MIP1 $\alpha$, MIP1 $\beta$, and MCP-1) associated with 


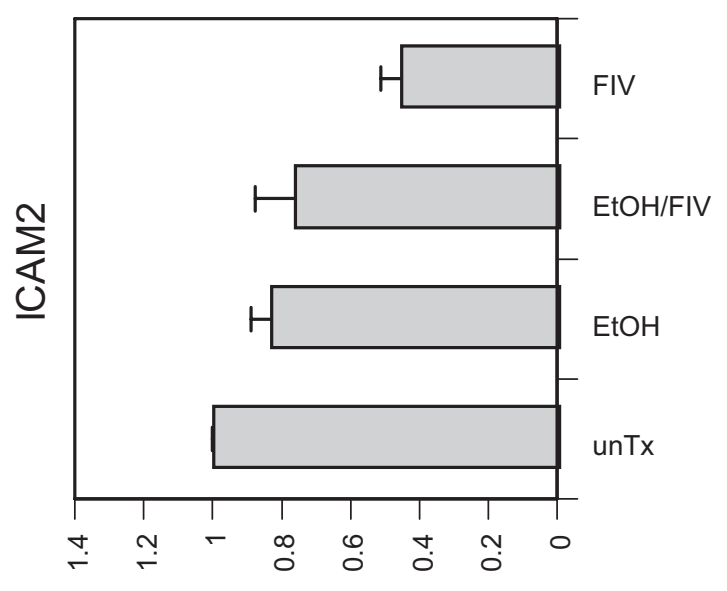

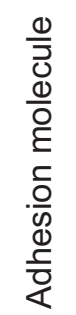

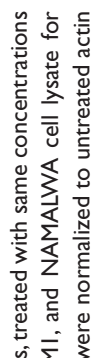
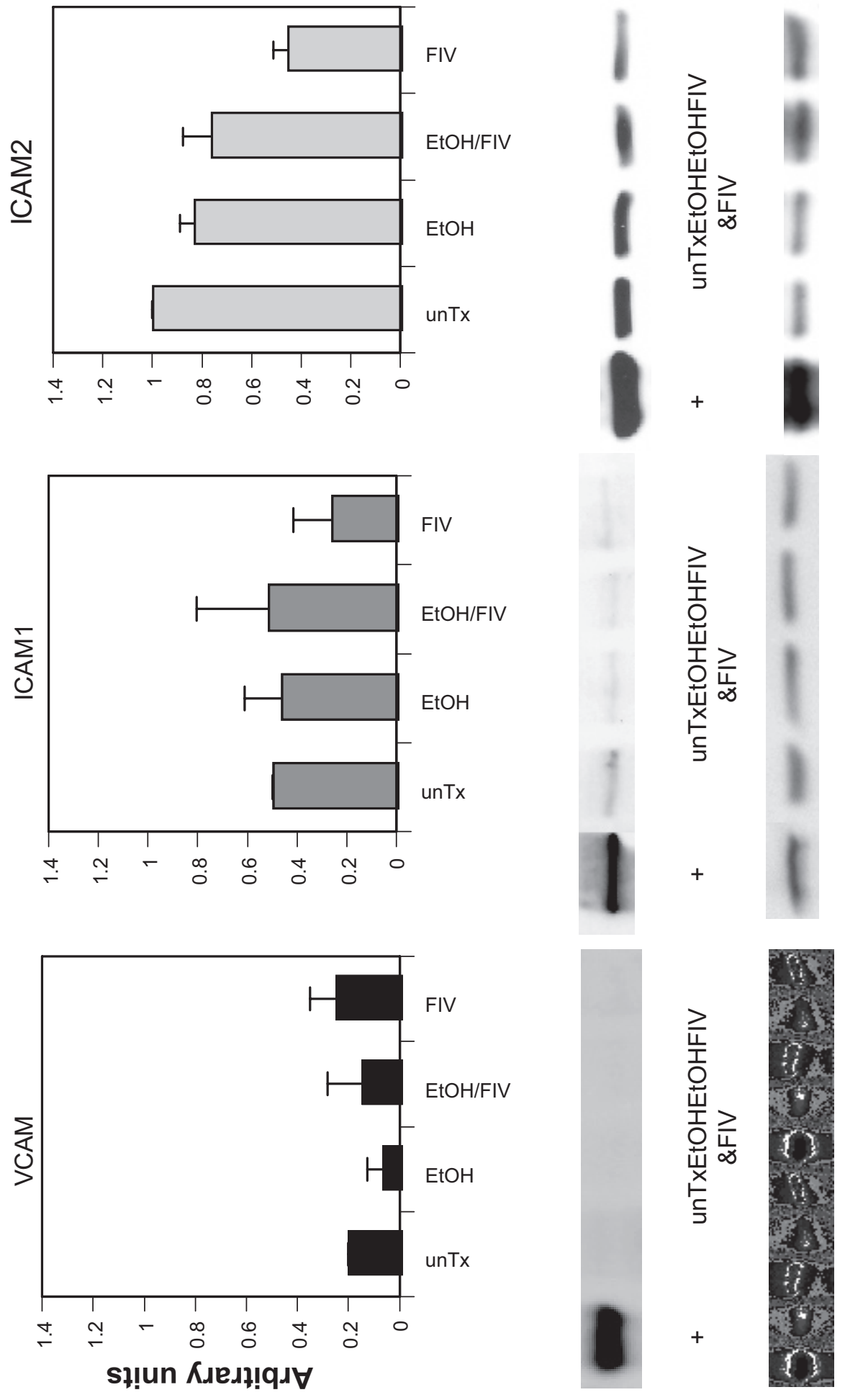

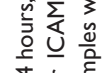

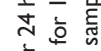

产遢

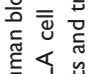

密

高家

है

要

爱

范它

总总

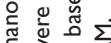

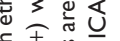

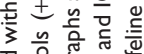

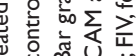

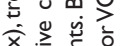

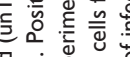

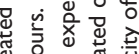

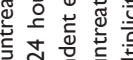

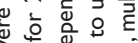

要

ن

势

要通

方资至过

Ư

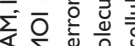

每

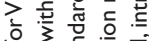

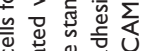

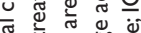

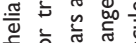

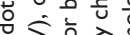

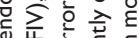

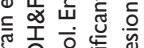

要

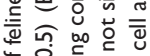

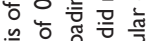

究

ल $\Sigma$ पू

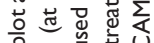

$0 \geq 0$

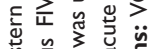

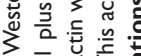

m 0 这

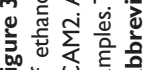

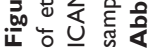




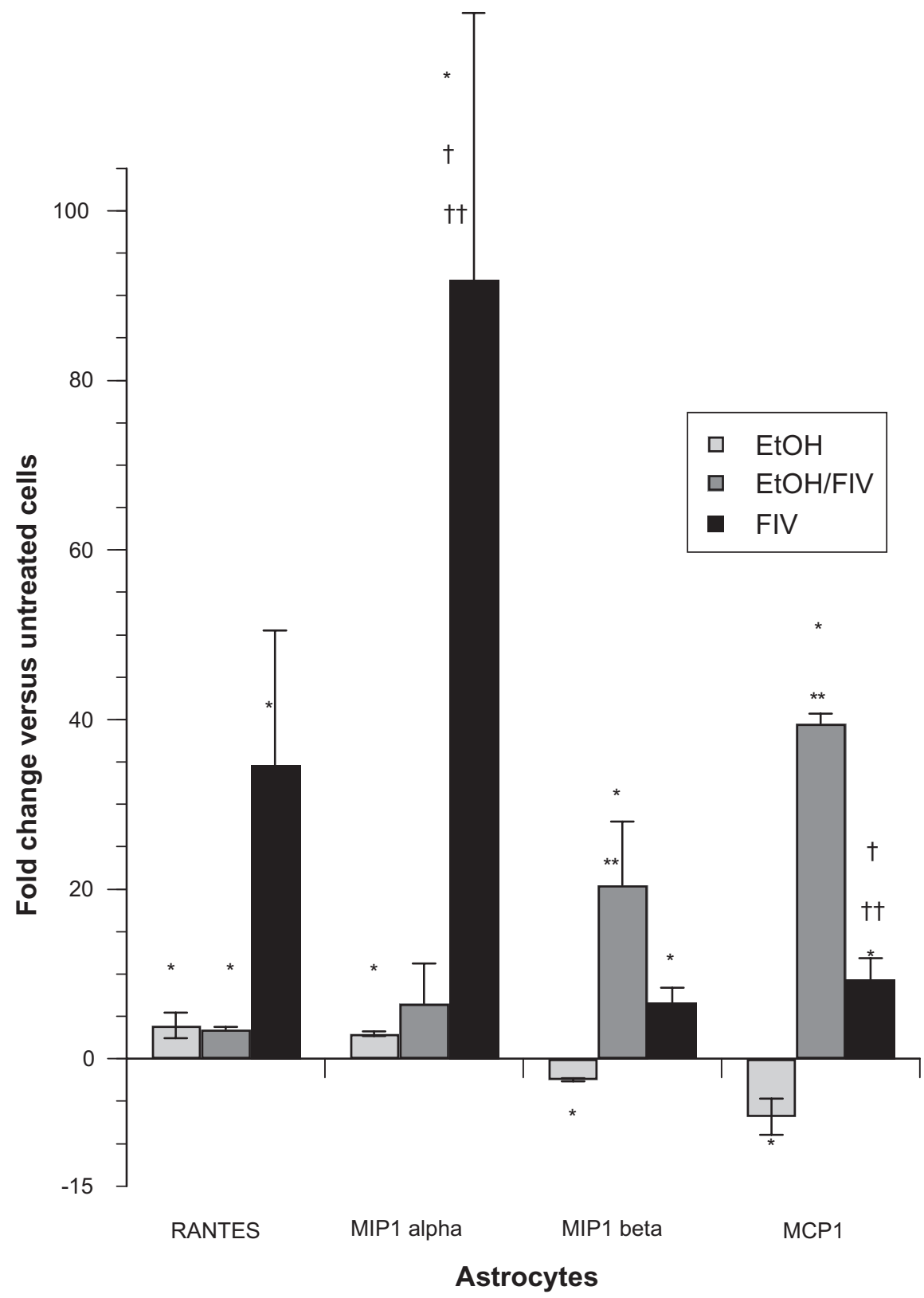

Figure 4 mRNA message levels of four chemokines in astrocytes. Cells were untreated (unTx), treated with ethanol (EtOH) to equal $0.08 \mathrm{mg} / \mathrm{dl}$ in human blood for 24 hours, treated with same concentrations of ethanol plus FIV (at MOI of 0.5 ) (EtOH\&FIV), or treated with MOI of 0.5 of FIV respectively, for 24 hours. Fold changes were indexed to untreated cells $\left(0\right.$ line). $* P<0.05$ of indicated bar compared to untreated cells. $* * P<0.05$ for ethanol and FIV treatment compared to ethanol alone. ${ }^{\star} P<0.05$ for FIV treatment compared to ethanol alone. ${ }^{t+P}<0.05$ for FIV treatment compared to ethanol and FIV treatment.

Abbreviations: RANTES, regulated upon activation, normal T expressed and presumably secreted; MIP, macrophage inflammatory protein; MCP, macrophage chemoattractant protein; FIV, feline immunodeficiency virus; $\mathrm{MOI}$, multiplicity of infection.

chemotaxis of PBMCs were also studied. Astrocytes (Figure 4) treated with ethanol demonstrated significant changes in message of all four chemokines compared to untreated cells. RANTES and MIP1 $\alpha$ were both increased slightly (3-4-fold) while MIP1 $\beta$ and MCP-1 were decreased 2-6-fold. These changes were small when contrasted to the effects of FIV where substantial increases were seen for each chemokine (RANTES, 35-fold; MIP1 $\alpha$, 92-fold; MIP1 $\beta$, 7-fold; MCP-1, 10-fold). Treatment with ethanol with FIV decreased RANTES and
MIP $1 \alpha$ and increased expression of MIP1beta and MCP-1 to 20 -fold and 38 -fold above controls, respectively.

Microglia generally demonstrated small or negligible changes in RANTES, MIP1 $\alpha$, MIP1 $\beta$, and MCP1 with any of the three treatments (Figure 5A) relative to untreated cells. MCP 1 was slightly increased by FIV and RANTES, MIP- $1 \alpha$ and MIP- $1 \beta$ decreased by ethanol.

Brain endothelial cells generally showed significant decreases in RANTES and MCP-1 messages and increases 
A

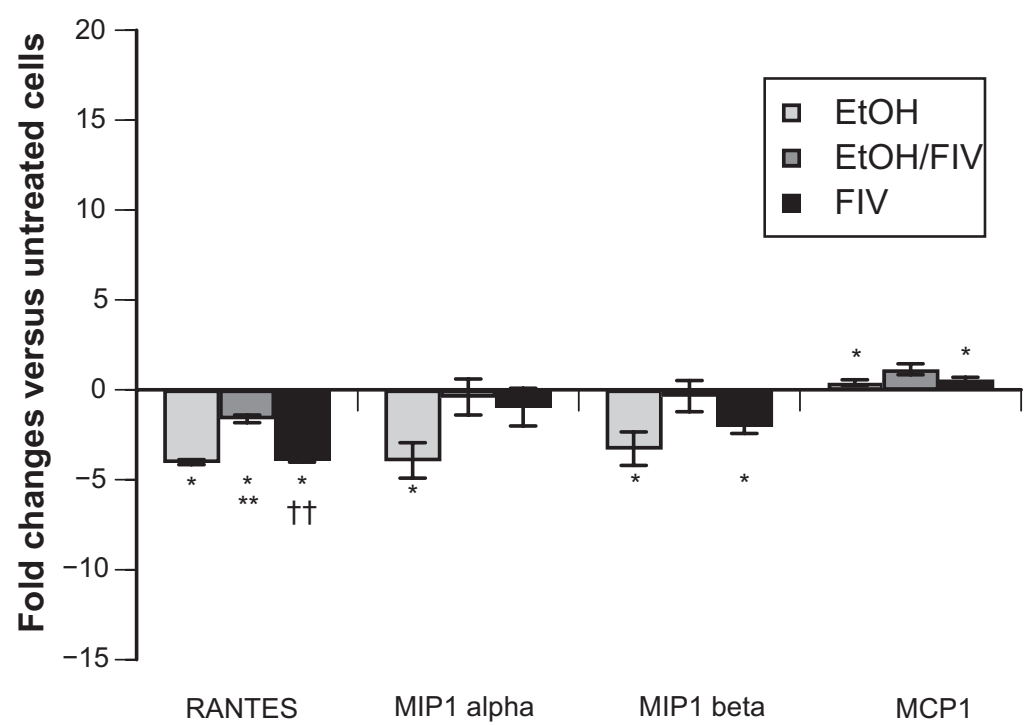

B

\section{Microglia}

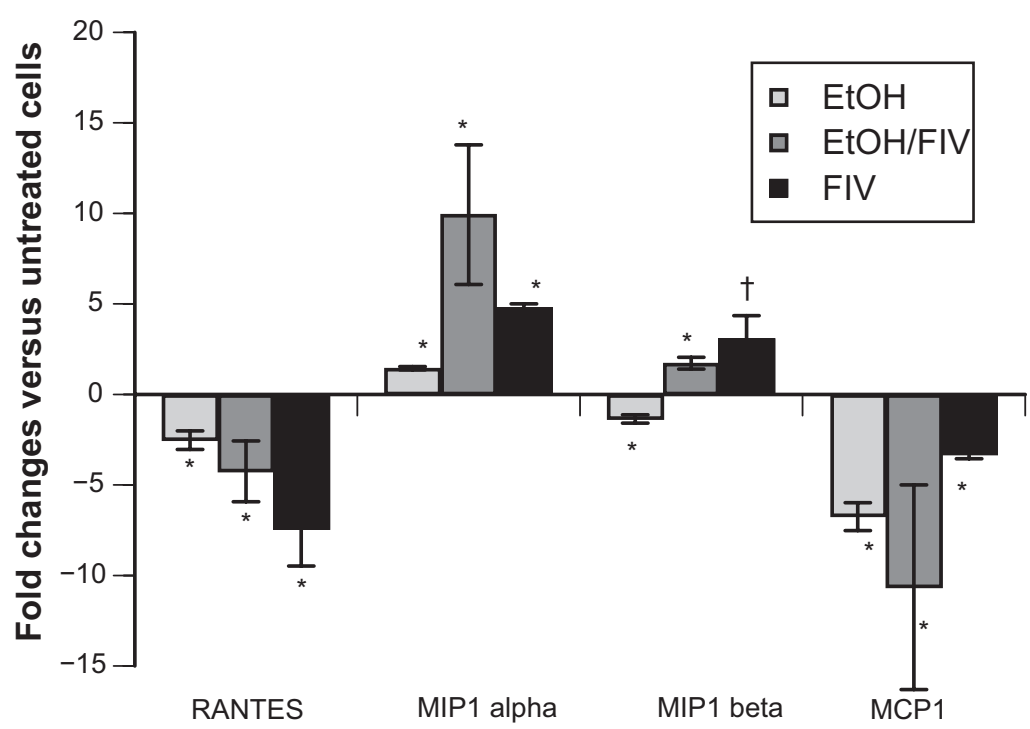

Brain Endothelial Cells

Figure 5 A) mRNA message levels of four chemokines in microglia and $\mathbf{B}$ ) brain endothelial cells. Cells were untreated (unTx), treated with ethanol (EtOH) to equal $0.08 \mathrm{mg} / \mathrm{dL}$ in human blood for 24 hours, treated with same concentrations of ethanol plus FIV (at MOI of 0.5 ) (EtOH\&FIV), or treated with MOI of 0.5 of FIV respectively, for 24 hours. Fold changes ${ }^{17}$ were indexed to untreated cells ( 0 line). $* P<0.05$ of indicated bar compared to untreated cells. **P $<0.05$ for ethanol and FIV treatment compared to ethanol alone. ${ }^{t} P<0.05$ for FIV treatment compared to ethanol alone. ${ }^{\dagger \dagger} P<0.05$ for FIV treatment compared to ethanol and FIV treatment.

Abbreviations: RANTES, regulated upon activation, normal T expressed and presumably secreted; MIP, macrophage inflammatory protein; MCP, macrophage chemoattractant protein; FIV, feline immunodeficiency virus; $\mathrm{MOI}$, multiplicity of infection.

in MIP1 $\alpha$ and $\beta$ message (Figure 5B) under all treatments compared to untreated cells. The magnitude of these changes was generally greater than that seen for microglia but much less than that seen for astrocytes.

\section{Discussion}

It has been hypothesized that abuse of drugs, including alcohol, increases the risk of CNS pathogenesis through a variety of mechanisms. Some investigators have suggested that alcohol use may lead to loss of inhibitions with increased high risk sex behaviors resulting in increased probability of HIV exposure(s). ${ }^{18-20}$ Other studies have indicated that alcohol abuse may independently increase the percentage of HIV+ patients with neurologic manifestations. ${ }^{1,5}$ These latter observations have raised questions about the specific impact of ethanol on HIV infection and neuropathogenesis. 
Various hypotheses have suggested that ethanol abuse could increase viral titers, result in an increase in immune cell trafficking or synergize with pathogenic mechanisms in the CNS. The possibility that ethanol might influence immune cell trafficking is particularly interesting since it may not only influence the penetration of infected cells but alter the CNS immune response to HIV. In addition, since it readily penetrates the blood-brain barrier, ethanol effects could be exerted centrally. Evidence in support of increased immune cell trafficking has derived, in part, from studies showing alcohol-related endothelial cell damage, increased permeability and increased expression of chemokine receptors which serve as co-receptors for HIV. ${ }^{21}$ Less work has been done to document changes in the transmigration of various immune cells across the endothelium during exposure to moderate concentrations of ethanol.

Since many cell types may be influenced by ethanol, we used an in vitro model that allowed assessment of the potential contribution of endothelial cells, astrocytes, or microglia. The contribution of each cell type to immune cell trafficking was determined by exposure to modest concentrations of ethanol to reflect a moderate level of abuse. In this investigation, PBMC adherence was significantly increased after treatment with ethanol for all combinations of cells but the presence/absence of astrocytes and microglia had no effect. The ethanol concentration was at modest physiologic levels, ${ }^{22,23} 80 \mathrm{mg} \%$, reflecting moderate chronic abuse. The results do not address possible changes under conditions of high acute abuse where blood concentrations may rise to $500 \mathrm{mg} \%$. FIV treatment alone did not significantly alter PBMC adherence as reported previously. ${ }^{15}$ The presence of microglia suppressed the ethanol-induced adherence of the PBMCs although levels were still above basal adherence.

As adherence is an early step in the trafficking mechanisms, this finding suggested that ethanol might enhance PBMC trafficking into the CNS. However, this possibility was not supported by the transmigration experiments. Such findings provide additional support that the control mechanisms for the different steps of trafficking are not directly linked, ie, the act of adherence does not automatically cause cellular transmigration. Treatment with either ethanol or ethanol in the presence of FIV generally resulted in markedly decreased numbers of transmigrated cells. This was in agreement with Saeed and colleagues ${ }^{10}$ where leukocyte transmigration was decreased in peripheral tissues after acute ethanol exposure. The lack of any synergism between alcohol and FIV further indicated that circulating virus does interact with ethanol to influence trafficking.
Penetration of PBMC subsets across the blood-brain barrier was similar to previous reports ${ }^{15}$ for untreated cells and FIV-treated cells indicating active regulation of immune cell transmigration in this paradigm. A positive effect of astrocytes on cell trafficking at the blood-brain barrier has been reported previously. ${ }^{15}$ While the mechanism of this is still not understood, it is clear that astrocytes can exert significant control over immune cell trafficking. In contrast, microglia are frequently seen to suppress the astrocyte effect. ${ }^{15,24}$ Thus, drugs of abuse have the potential to influence immune cell trafficking through a variety of mechanisms involving several different cell types. Exposure to ethanol eliminated these interactions suggesting an early block of transmigration.

The lack of transmigration with ethanol treatment was corroborated by a failure to see increases in supportive mechanisms. Conditions known to support immune cell trafficking such as exposure to cytokines will upregulate adhesion molecules, such as VCAM, ICAM1, and E-selectin in endothelial cells. ${ }^{25-28}$ However, none of the three common adhesion molecules that we examined showed increased protein expression after ethanol or ethanol plus FIV. This finding generally agreed with the observations of Saeed and colleagues $^{10}$ in which ethanol alone decreased total VCAM and E-selectin expression on peripheral endothelial cells. It was also shown that FIV alone did not increase expression of total protein or message of ICAM1, ICAM2, or VCAM in feline BECs. ${ }^{24}$ On the contrary ICAM2, which was most abundantly expressed, was decreased about 50\% following FIV treatment of the endothelial cells as had been reported previously. ${ }^{24}$ These observations suggested that the PBMC adherence seen in coculture was either the result of some other adhesion molecule(s) or perhaps a nonspecific interaction.

Changes in chemokine message supported previous findings highlighting the important role of astrocytes and microglia in the transmigration of PBMCs. Again the interpretation is limited by a lack of feline-specific reagents suitable for the analysis of chemokine protein. The highest levels of transmigration for CD4 T cells, CD8 T cells, monocytes and $B$ cells were associated with the presence of astrocytes. FIV treatment further increased the transmigration of monocytes. Astrocytes expressed the highest level of chemokine mRNA and showed the greatest increases following exposure to FIV, which may explain their prominent effects on immune cell transmigration. Ethanol alone showed low increases or even decreases in cytokine message in the astrocytes. However, the combination of ethanol and FIV resulted in a significant increase in MIP1 beta and MCP-1 mRNA. This synergism 
by ethanol failed to translate to an increase in transmigration. Together these observations suggest that the general suppression of transmigration by ethanol was most likely due to a failure at early stages of transmigration.

Thus, the possibility that moderate alcohol abuse might result in more effective $\mathrm{PBMC}$ transmigration in infected or uninfected individuals was not supported by this study. Instead alcohol suppressed transmigration of all PBMC subsets in the vast majority of configurations. This suppression of trafficking could be interpreted in different ways. On the one hand, it could restrict entry of infected cells into the CNS and slow early disease progression. On the other hand, inefficient trafficking of immune cells may eliminate important mechanisms needed for control of viral replication and clearance of virus from the CNS which appears to be an early protective response. ${ }^{29} \mathrm{~A}$ further consideration is that the alcohol level utilized was at the legal limit for intoxication and it remains possible that higher levels and chronic administration for greater than 24 hours may differentially affect CNS trafficking. For example, opposite effects of ethanol have been seen with in vitro toxicity studies with low levels protecting against gp120 toxicity and high doses synergizing with gp120 toxicity. Our findings do not address effects in peripheral systems nor do they exclude alterations in the trafficking of cells and virus at the choroid plexus. ${ }^{30,31}$

Overall, attempts to understand the role of ethanol abuse in HIV neuropathogenesis need to factor in the potential effects of decreased immune cell trafficking as well as possible changes in chemokine signaling by astrocytes. More work is clearly needed to fully appreciate the various interactions of ethanol in neuroinflammatory and neuroprotective processes.

\section{Acknowledgments}

This investigation was supported by funds from National Institutes of Health (MH63646 and AI47749), and the state of North Carolina. The authors report no conflicts of interest in this work.

\section{References}

1. Pfefferbaum A, Adalsteinsson E, Sullivan EV. Cortical NAA deficits in HIV infection without dementia: influence of alcoholism comorbidity. Neuropsychopharmacology. 2005;30(7):1392-1399.

2. Conigliaro J, Gordon AJ, McGinnis KA, Rabeneck L, Justice AC. How harmful is hazardous alcohol use and abuse in HIV infection: do health care providers know who is at risk? J Acquir Immune Defic Syndr. 2003;33(4):521-525.

3. Zheng J, Yang OO, Xie Y, Campbell R, Chen IS, Pang S. Ethanol stimulation of HIV infection of oral epithelial cells. J Acquir Immune Defic Syndr. 2004;37(4):1445-1453.
4. Tyor WR, Middaugh LD. Do alcohol and cocaine abuse alter the course of HIV-associated dementia complex? J Leukoc Biol. 1999;65(4): 475-481.

5. Meyerhoff DJ. Effects of alcohol and HIV infection on the central nervous system. Alcohol Res Health. 2001;25(4):288-298.

6. Poonia B, Nelson S, Bagby GJ, Zhang P, Quniton L, Veazey RS. Chronic alcohol consumption results in higher simian immunodeficiency virus replication in mucosally inoculated rhesus macaques. AIDS Res Hum Retroviruses. 2005;21(10):863-868.

7. Kumar R, Perez-Casanova AE, Tirado G, et al. Increased viral replication in simian immunodeficiency virus/simian-HIV-infected macaques with self-administering model of chronic alcohol consumption. J Acquir Immune Defic Syndr. 2005;39(4):386-390.

8. Liu X, Zha J, Nishitani J, Chen H, Zack JA. HIV-1 infection in peripheral blood lymphocytes (PBLs) exposed to alcohol. Virology. 2003; 307(1):37-44.

9. Witte MH, Borgs P, Way DL, Ramirez G Jr, Witte CL, Bernas MJ. AIDS, alcohol, endothelium, and immunity. Alcohol. 1994;11(2):91-97.

10. Saeed RW, Varma S, Peng T, Tracey KJ, Sherry B, Metz CN. Ethanol blocks leukocyte recruitment and endothelial cell activation in vivo and in vitro. J Immunol. 2004;173(10):6376-6383.

11. Kono H, Uesugi T, Froh M, Rusyn I, Bradford BU, Thurman RG. ICAM-1 is involved in the mechanism of alcohol-induced liver injury: studies with knockout mice. Am J Physiol Gastrointest Liver Physiol. 2001;280(6):G1289-G1295.

12. Sacanella E, Estruch R, Badia E, Fernandez-Sola J, Nicolas JM, UrbanoMarquez A. Chronic alcohol consumption increases serum levels of circulating endothelial cell/leucocyte adhesion molecules E-selectin and ICAM-1. Alcohol Alcohol. 1999;34(5):678-684.

13. Dingle GA, Oei TP. Is alcohol a cofactor of HIV and AIDS? Evidence from immunological and behavioral studies. Psychol Bull. 1997;122(1): 56-71.

14. Bolton R, Vincke J, Mak R, Dennehy E. Alcohol and risky sex: in search of an elusive connection. Med Anthropol. 1992;14(2-4):323-363.

15. Hudson LC, Bragg DC, Tompkins MB, Meeker RB. Astrocytes and microglia differentially regulate trafficking of lymphocyte subsets across brain endothelial cells. Brain Res. 2005;1058:148-160.

16. Tompkins MB, Nelson PD, English RV, Novotney C. Early events in the immunopathogenesis of feline retrovirus infections. J Am Vet Med Assoc. 1991;199:1311-1315.

17. Winer J, Jung CK, Shackel I, Williams PM. Development and validation of real-time quantitative reverse transcriptase-polymerase chain reaction for monitoring gene expression in cardiac myocytes in vitro. Anal Biochem. 1999;270(1):41-49.

18. Mbulaiteye SM, Ruberantwari A, Nakiyingi JS, Carpenter LM, Kamali A, Whitworth JA. Alcohol and HIV: a study among sexually active adults in rural southwest Uganda. Int J Epidemiol. 2000;29(5):911-915.

19. Stueve A, O’Donnell LN. Early alcohol initiation and subsequent sexual and alcohol risk behaviors among urban youths. Am J Public Health. 2005;95(5):887-893.

20. Wang Y, Watson RR. Is alcohol consumption a cofactor in the development of acquired immunodeficiency syndrome? Alcohol. 1995;12(2):105-109.

21. Shiu C, Barbier E, Di Cello F, Choi HJ, Stins M. HIV-1 gp120 as well as alcohol affect blood-brain barrier permeability and stress fiber formation: involvement of reactive oxygen species. Alcohol Clin Exp Res. 2007;31(1):130-137.

22. Huang CM, Huang RH. Ethanol inhibits the sensory responses of cerebellar granule cells in anesthetized cats. Alcohol Clin Exp Res. 2007;31(2):336-344.

23. Agapejev S, Vassilieff I, Curi PR. Alcohol levels in cerebrospinal fluid and blood samples from patients under pathological conditions. Acta Neurol Scand. 1992;86(5):496-500.

24. Hudson LC, Tompkins MB, Meeker RB. Endothelial cell suppression of peripheral blood mononuclear cell trafficking in vitro during acute exposure to feline immunodeficiency virus. Cell Tissue Res. 2008;334(1):55-65. 
25. Wong D, Dorovini-Zis K. Upregulation of intercellular adhesion molecule-1 (ICAM-1) expression in primary cultures of human brain microvessel endothelial cells by cytokines and lipopolysaccharide. J Neuroimmunol. 1992;39(1-2):11-21.

26. Wong D, Dorovini-Zis K. Expression of vascular cell adhesion molecule-1 (VCAM-1) by human brain microvessel endothelial cells in primary culture. Microvasc Res. 1995;49(3):325-339.

27. Wong D, Dorovini-Zis K. Regualtion by cytokines and lipopolysaccharide of E-selectin expression by human brain microvessel endothelial cells in primary culture. J Neuropathol Exp Neurol. 1996;55(2):225-235.

28. Wong D, Prameya R, Dorovini-Zis K. In vitro adhesion and migration of T lymphocytes across monolayers of human brain microvessel endothelial cells: regulation by ICAM-1, VCAM-1, E-selectin and PECAM-1. J Neuropathol Exp Neurol. 1999;58(2):138-152.
29. Ryan G, Grimes T, Brankin B, et al. Neuropathology associated with feline immunodeficiency virus infection highlights prominent lymphocyte trafficking through both the blood-brain and blood-choroid plexus barriers. J Neurovirol. 2005;11(4):337-345.

30. Liu P, Hudson LC, Tompkins MB, et al. Cerebrospinal fluid is an efficient route for establishing brain infection with feline immunodeficiency virus and transferring infectious virus to the periphery. J Neurovirol. 2006;12(4):294-306

31. Liu P, Hudson LC, Tompkins MB, Vahlenkamp TW, Meeker RB. Compartmentalization and evolution of feline immunodeficiency virus between the central nervous system and periphery following intracerebroventricular or systemic inoculation. J Neurovirol. 2006;12(4): 307-321.

\section{Publish your work in this journal}

HIV/AIDS - Research and Palliative Care is an international, peerreviewed open-access journal focusing on advances in research in HIV, its clinical progression and management options including antiviral treatment, palliative care and public healthcare policies to control viral spread. The journal welcomes original research, basic science, clinical \& epidemiological studies, reviews \& evaluations, expert opinion \& commentary, case reports \& extended reports. The manuscript management system is completely online and includes a very quick and fair peer-review system. Visit http://www.dovepress.com/ testimonials.php to read real quotes from published authors. 